\title{
Integrable dynamics of a discrete curve and the Ablowitz-Ladik hierarchy *
}

\author{
Adam Doliwa \\ Institute of Theoretical Physics, Warsaw University \\ ul. Hoża 69, 00-681 Warsaw, Poland \\ Paolo Maria Santini \\ Dipartimento di Fisica, Università di Roma "La Sapienza" \\ and INFN, Sezione di Roma \\ P.le Aldo Moro 2, I-00185 Roma, Italy
}

\begin{abstract}
We show that the following elementary geometric properties of the motion of a discrete (i.e. piecewise linear) curve select the integrable dynamics of the Ablowitz-Ladik hierarchy of evolution equations: i) the set of points describing the discrete curve lie in the sphere $S^{3}$; ii) the distance between any two subsequent points does not vary in time; iii) the dynamics does not depend explicitly on the radius of the sphere. These results generalize to a discrete context our previous work on continuous curves [1].
\end{abstract}

${ }^{*}$ This work was supported by the 1993 agreement between Rome and Warsaw Universities; by the grant 2-0168-91-01 KBN and by the INFN. 


\section{Integrable discrete dynamics and geometry}

During the last two decades many important classes of nonlinear equations have been solved. These integrable nonlinear equations appear in very different forms: they are ordinary differential equations (ODE's) (autonomous and not) [2], partial differential equations (PDE's) [3], algebraic and functional equations 酒. It was also possible to construct integrable discrete analogues of all the important nonlinear

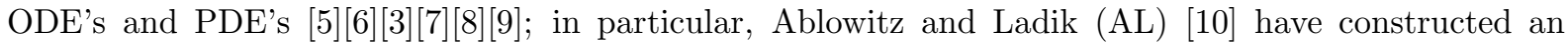
integrable discrete analogue of the AKNS hierarchy [11], associated with the following discrete eigenvalue problem

$$
\left[\begin{array}{l}
\phi_{k+1}^{(1)} \\
\phi_{k+1}^{(2)}
\end{array}\right]=\left[\begin{array}{cc}
\zeta & q_{k} \\
r_{k} & \zeta^{-1}
\end{array}\right]\left[\begin{array}{c}
\phi_{k}^{(1)} \\
\phi_{k}^{(2)}
\end{array}\right],
$$

where the complex fields $q_{k}$ and $r_{k}$ depend on the discrete variable $k(k \in \mathbf{Z})$, and the complex parameter $\zeta$ plays the role of spectral parameter.

The interesting connections between the differential geometry of submanifolds and integrable nonlinear PDE's, which have been shown many times throught the years (the literature on this subject is very large and we refer to our previous paper [1] for a historical review), can also be found at a discrete level. For instance, discrete pseudospherical surfaces and the discrete analogues of constant mean curvature surfaces are described by integrable discrete analogues of the sine-Gordon (SG) 12 and sinh-Gordon equations [13. Such discretizations were found by adapting the main geometric properties of the continuous surfaces to a discrete level.

In a recent paper [1] we have shown that the following three elementary geometric properties of the motion of a curve select, among all possible dynamics, integrable dynamics:

Property 1. The motion of the curve takes place in the $N$-dimensional sphere of radius $R$, denoted by $S^{N}(R), N>1$.

Property 2. The curve does not stretch during the motion.

Property 3. The dynamics of the curve does not depend explicitly on the radius $R$.

These dynamics are described by integrable commuting nonlinear evolution equations in $1+1$ (one spatial and one temporal) dimensions for the geodesic curvatures of the curve. More precisely, for $N=2$ we have obtained the modified Korteweg - de Vries $(\mathrm{mKdV})$ hierarchy for the curvature $\kappa$. For $N=3$ we have obtained a hierarchy of evolution equations for the geodesic curvature $\kappa=\kappa_{1}$ and torsion $\tau=\kappa_{2}$, which can be transformed, through the Hasimoto transformation, into the nonlinear Schrödinger (NLS) hierarchy. For $N>3$ we have obtained integrable multicomponent generalizations of the above hierarchies. Also integrable evolution equations "with sources" (with singular dispersion relation) have been selected by Properties 1-3.

The goal of this paper is to construct large classes of geometrically meaningful discrete evolution equations applying the above Properties 1-3 to the case of a discrete curve, where by discrete curve we mean just a sequence of points. The obvious discrete generalization of Properties 1-3 is the following.

Property 1'. The motion of the discrete curve takes place in the $N$-dimensional sphere $S^{N}(R)(N>1)$ of radius $R$.

Property 2'. The distance between any two subsequent points does not vary in time.

Property 3'. The dynamics of the discrete curve does not depend explicitly on the radius $R$.

We will consider the cases $N=2$ and $N=3$, showing that Properties 1'-3' generate the AL hierarchy of evolution equations. In particular we will prove that Property 1' for $N=2$ and $N=3$ is equivalent to the spectral problem (1) in the reductions $r_{k}=-q_{k} \in \mathbf{R}$ and $r_{k}=-\bar{q}_{k}$ respectively.

In this paper we concentrate our attention on continuous evolutions, i.e. time $t$ is a continuous variable; we postpone to a subsequent work the study of integrable discrete dynamics of a curve. 


\section{The motion of a discrete curve on $S^{2}(R)$}

The problem of a curve on $S^{2}$ is a special of case of that of a curve in $S^{3}$, but because of its pedagogical importance we have decided to treat it first.

\subsection{The discrete curve on $S^{2}(R)$}

Generalizing the problem of a discrete curve on the plane (see, eg. [14]), we derive now the set of equations which describe a discrete curve on $S^{2}(R)$. We indicate by $\overrightarrow{\mathbf{r}}_{k},(k \in \mathbf{Z})$ the position vector of the $k^{\text {th }}$ point of the sequence pointing from the centre of the sphere. The unit vector in the direction of $\overrightarrow{\mathbf{r}}_{k}$ is denoted by $\hat{\mathbf{r}}_{k}$. The oriented plane $\pi_{k}$ is given by the ordered pair $\left(\overrightarrow{\mathbf{r}}_{k}, \overrightarrow{\mathbf{r}}_{k+1}\right)$. By $\hat{\mathbf{t}}_{k}\left(\hat{\mathbf{t}}_{k}^{\prime}\right)$ we denote the unit vector of the plane $\pi_{k}$ orthogonal to $\hat{\mathbf{r}}_{k}\left(\hat{\mathbf{r}}_{k+1}\right)$ with the direction given by the orientation of the plane $\pi_{k}$. Both $\hat{\mathbf{t}}_{k}$ and $\hat{\mathbf{t}}_{k}^{\prime}$ are tangent (in the points $\overrightarrow{\mathbf{r}}_{k}$ and $\overrightarrow{\mathbf{r}}_{k+1}$ correspondingly) to the big circle passing through the points $\overrightarrow{\mathbf{r}}_{k}$ and $\overrightarrow{\mathbf{r}}_{k+1}$.

In the following we assume that the segments of geodesics which join subsequent points have the same length $\Delta$ for each pair of the sequence. The angle $\alpha$ is one-half of the angle $\lambda \Delta$ (where $\lambda=1 / R$ ) between the vectors $\overrightarrow{\mathbf{r}}_{k}$ and $\overrightarrow{\mathbf{r}}_{k+1}$ :

$$
\alpha=\Delta /(2 R) .
$$

Together with the basis $\left(\hat{\mathbf{r}}_{k}, \hat{\mathbf{t}}_{k}\right)$ of the plane $\pi_{k}$ we will use also the new basis $\left(\hat{\mathbf{r}}_{k}^{\prime}, \hat{\mathbf{a}}_{k}\right)$ obtained from the old one by rotation of the angle $\alpha$

$$
\begin{aligned}
\hat{\mathbf{r}}_{k}^{\prime} & =\hat{\mathbf{r}}_{k} \cos \alpha-\hat{\mathbf{t}}_{k} \sin \alpha \\
\hat{\mathbf{a}}_{k} & =\hat{\mathbf{t}}_{k} \cos \alpha+\hat{\mathbf{r}}_{k} \sin \alpha
\end{aligned}
$$

We remark that

$$
\hat{\mathbf{a}}_{k}=\frac{\overrightarrow{\mathbf{r}}_{k+1}-\overrightarrow{\mathbf{r}}_{k}}{\left|\overrightarrow{\mathbf{r}}_{k+1}-\overrightarrow{\mathbf{r}}_{k}\right|}
$$

We define the normal vector $\hat{\mathbf{n}}_{k}$ by $\hat{\mathbf{n}}_{k}=\hat{\mathbf{r}}_{k} \times \hat{\mathbf{t}}_{k}$, where the cross-product $\times$ is consistent with the given orientation of the ambient $\mathbf{R}^{3}$. The three vectors $\left(\hat{\mathbf{r}}_{k}, \hat{\mathbf{t}}_{k}, \hat{\mathbf{n}}_{k}\right)$ form the orthonormal basis of $\mathbf{R}^{3}$ in the point $\overrightarrow{\mathbf{r}}_{k}$ of the discrete curve.

The oriented tangent plane $\tau_{k}$ is given by the ordered pair of vectors $\left(\hat{\mathbf{t}}_{k}, \hat{\mathbf{n}}_{k}\right)$. Since $\hat{\mathbf{r}}_{k} \perp \tau_{k}$ and $\hat{\mathbf{r}}_{k} \perp \hat{\mathbf{t}}_{k-1}^{\prime}$, then $\hat{\mathbf{t}}_{k-1}^{\prime} \in \tau_{k}$. By $\varphi_{k-1}\left(\left|\varphi_{k-1}\right|<\pi\right)$ we denote the angle between $\hat{\mathbf{t}}_{k-1}^{\prime}$ and $\hat{\mathbf{t}}_{k}$ measured according to the orientation of the plane $\tau_{k} ; \varphi_{k-1}$ is the angle of geodesic curvature. We remark that $\varphi_{k-1}$ is also the angle between the planes $\pi_{k-1}$ and $\pi_{k}$.

In the continuous limit: $\Delta \rightarrow 0$ and $\Delta k \rightarrow s$, where $s$ is the arc-length parameter of the corresponding curve, the angle of geodesic curvature reduces to the familiar geodesic curvature $\kappa(s)$ in the following way:

$$
\frac{\varphi_{k}}{\Delta} \stackrel{\Delta \rightarrow 0}{\longrightarrow} \kappa(s)
$$

The transition from the basis $\left(\hat{\mathbf{r}}_{k}, \hat{\mathbf{t}}_{k}, \hat{\mathbf{n}}_{k}\right)$ to the basis $\left(\hat{\mathbf{r}}_{k+1}, \hat{\mathbf{t}}_{k+1}, \hat{\mathbf{n}}_{k+1}\right)$ can be obtained by the superposition of two rotations:

i) rotation in the plane $\pi_{k}$ of the angle $\lambda \Delta=2 \alpha$

$$
\left(\hat{\mathbf{r}}_{k}, \hat{\mathbf{t}}_{k}, \hat{\mathbf{n}}_{k}\right) \longrightarrow\left(\hat{\mathbf{r}}_{k+1}, \hat{\mathbf{t}}_{k}^{\prime}, \hat{\mathbf{n}}_{k}\right)
$$


ii) rotation in the plane $\tau_{k+1}$ of the angle $\varphi_{k}$

$$
\left(\hat{\mathbf{r}}_{k+1}, \hat{\mathbf{t}}_{k}^{\prime}, \hat{\mathbf{n}}_{k}\right) \longrightarrow\left(\hat{\mathbf{r}}_{k+1}, \hat{\mathbf{t}}_{k+1}, \hat{\mathbf{n}}_{k+1}\right) .
$$

The full rotation can be written in matrix form as:

$$
\left[\begin{array}{c}
\hat{\mathbf{r}}_{k+1} \\
\hat{\mathbf{t}}_{k+1} \\
\hat{\mathbf{n}}_{k+1}
\end{array}\right]=\left[\begin{array}{ccc}
\cos (\lambda \Delta) & \sin (\lambda \Delta) & 0 \\
-\cos \varphi_{k} \sin (\lambda \Delta) & \cos \varphi_{k} \cos (\lambda \Delta) & \sin \varphi_{k} \\
\sin \varphi_{k} \sin (\lambda \Delta) & -\sin \varphi_{k} \cos (\lambda \Delta) & \cos \varphi_{k}
\end{array}\right]\left[\begin{array}{c}
\hat{\mathbf{r}}_{k} \\
\hat{\mathbf{t}}_{k} \\
\hat{\mathbf{n}}_{k}
\end{array}\right]
$$

\subsection{The spinor representation of the linear problem}

To obtain the $2 \times 2$ version of the linear problem (6) we use the standard representation of rotations in $\mathbf{R}^{3}$ in terms of $S U(2)$-valued matrices [15. From this point of view $\mathbf{R}^{3}$ can be considered as the space spanned by the matrices $\varepsilon_{1}=i \sigma_{2}, \varepsilon_{2}=-i \sigma_{1}, \varepsilon_{3}=i \sigma_{3}$ (here $\sigma_{l}$ denote the standard Pauli matrices):

$$
\overrightarrow{\mathbf{v}} \in \mathbf{R}^{3} \Leftrightarrow \sigma(\overrightarrow{\mathbf{v}})=\sum_{l=1}^{3} \mathrm{v}^{l} \varepsilon_{l} ;\|\overrightarrow{\mathbf{v}}\|^{2}=\operatorname{det} \sigma(\overrightarrow{\mathbf{v}}),
$$

where $\mathrm{v}^{l}$ are the Cartesian coordinates of the vector $\overrightarrow{\mathbf{v}}$. We recall that any rotation $O$ in $\mathbf{R}^{3}$ can be represented by a matrix $S \in S U(2)$ by the formula

$$
\overrightarrow{\mathbf{w}}=O \overrightarrow{\mathbf{v}} \Leftrightarrow \sigma(\overrightarrow{\mathbf{w}})=S^{-1} \sigma(\overrightarrow{\mathbf{v}}) S .
$$

Suppose we are given a sequence of orthonormal frames $\mathcal{F}_{k}=\left(\mathbf{e}_{k}, \mathbf{f}_{k}, \mathbf{g}_{k}\right), k \in \mathbf{Z}$, and the corresponding matrices $S_{k}$

$$
\mathcal{F}_{k}=S_{k}^{-1} \mathcal{E} S_{k},
$$

where $\mathcal{E}=\left(\varepsilon_{1}, \varepsilon_{2}, \varepsilon_{3}\right)$. When matrices $A_{k}$ are defined by the relation

$$
S_{k+1}=A_{k} S_{k}
$$

then they satisfy also

$$
S_{k} \mathcal{F}_{k+1} S_{k}^{-1}=A_{k}^{-1} \mathcal{E} A_{k}
$$

For example, the matrix

$$
A_{k}=\left[\begin{array}{cc}
\mathrm{e}^{i \alpha} & 0 \\
0 & \mathrm{e}^{-i \alpha}
\end{array}\right]
$$

represents the rotation in the plane $\left(\mathbf{e}_{k}, \mathbf{f}_{k}\right)$ of the angle $2 \alpha$, and the matrix

$$
A_{k}=\left[\begin{array}{cc}
\cos (\varphi / 2) & \sin (\varphi / 2) \\
-\sin (\varphi / 2) & \cos (\varphi / 2)
\end{array}\right]
$$

represents the rotation in the plane $\left(\mathbf{f}_{k}, \mathbf{g}_{k}\right)$ of the angle $\varphi$.

When the sequence of orthonormal frames is the sequence of the Frenet frames in the points of the discrete curve: $\mathcal{F}_{k}=\left(\hat{\mathbf{r}}_{k}, \hat{\mathbf{t}}_{k}, \hat{\mathbf{n}}_{k}\right)$, then the corresponding spin matrices $S_{k}$ are subjected to the relation

$$
\begin{aligned}
S_{k+1} & =\left[\begin{array}{cc}
\mathrm{e}^{i \alpha} \cos \left(\varphi_{k} / 2\right) & \mathrm{e}^{-i \alpha} \sin \left(\varphi_{k} / 2\right) \\
-\mathrm{e}^{i \alpha} \sin \left(\varphi_{k} / 2\right) & \mathrm{e}^{-i \alpha} \cos \left(\varphi_{k} / 2\right)
\end{array}\right] S_{k} \\
& =\frac{1}{\sqrt{1+q_{k}^{2}}}\left[\begin{array}{cc}
\zeta & \zeta^{-1} q_{k} \\
-\zeta q_{k} & \zeta^{-1}
\end{array}\right] S_{k}=: A_{k} S_{k}
\end{aligned}
$$


with

$$
q_{k}=\tan \left(\varphi_{k} / 2\right), \quad \zeta=\mathrm{e}^{i \alpha} .
$$

We remark that this spectral problem is equivalent to the AL spectral problem

$$
\tilde{S}_{k+1}=\left[\begin{array}{cc}
\zeta & q_{k} \\
-q_{k} & \zeta^{-1}
\end{array}\right] \tilde{S}_{k}
$$

in the reduction $r_{k}=-q_{k} \in \mathbf{R}$. To show this we relax the (unessential) condition $\operatorname{det} S_{k}=1$ to remove the $\cos \left(\varphi_{k} / 2\right)$ diagonal term and apply a gauge transformation which corresponds to the change of the basis of the plane $\pi_{k}$ from $\left(\hat{\mathbf{r}}_{k}, \hat{\mathbf{t}}_{k}\right)$ to $\left(\hat{\mathbf{r}}_{k}^{\prime}, \hat{\mathbf{a}}_{k}\right): \mathcal{F}_{k}=\left(\hat{\mathbf{r}}_{k}, \hat{\mathbf{t}}_{k}, \hat{\mathbf{n}}_{k}\right) \rightarrow \tilde{\mathcal{F}}_{k}=\left(\hat{\mathbf{r}}_{k}^{\prime}, \hat{\mathbf{a}}_{k}, \hat{\mathbf{n}}_{k}\right)$. Then

$$
\tilde{\mathcal{F}}_{k}=\tilde{S}_{k}^{-1} \mathcal{E} \tilde{S}_{k}
$$

where

$$
\tilde{S}_{k}=c_{k}\left[\begin{array}{cc}
\mathrm{e}^{i \alpha / 2} & 0 \\
0 & \mathrm{e}^{-i \alpha / 2}
\end{array}\right] S_{k}\left[\begin{array}{cc}
\mathrm{e}^{-i \alpha / 2} & 0 \\
0 & \mathrm{e}^{i \alpha / 2}
\end{array}\right]
$$

and

$$
c_{k+1}=\frac{c_{k}}{\cos \left(\varphi_{k} / 2\right)}=c_{k} \sqrt{1+q_{k}^{2}} .
$$

\subsection{The integrable motions on $S^{2}(R)$}

Since the motion takes place on the sphere, the velocity field is tangent to it:

$$
\overrightarrow{\mathbf{r}}_{k, t}=\tilde{V}_{k} \hat{\mathbf{t}}_{k}+\tilde{U}_{k} \hat{\mathbf{n}}_{k} .
$$

Futhermore the evolution of the Frenet frame is described by an antisymmetric matrix:

$$
\left[\begin{array}{c}
\hat{\mathbf{r}}_{k} \\
\hat{\mathbf{t}}_{k} \\
\hat{\mathbf{n}}_{k}
\end{array}\right]_{, t}=\left[\begin{array}{ccc}
0 & \lambda \tilde{V}_{k} & \lambda \tilde{U}_{k} \\
-\lambda \tilde{V}_{k} & 0 & A_{k} \\
-\lambda \tilde{U}_{k} & -A_{k} & 0
\end{array}\right]\left[\begin{array}{c}
\hat{\mathbf{r}}_{k} \\
\hat{\mathbf{t}}_{k} \\
\hat{\mathbf{n}}_{k}
\end{array}\right]
$$

or equivalently, by the evolution of the matrix $S_{k}$ :

$$
S_{k, t}=\frac{1}{2}\left[\begin{array}{cc}
i \lambda \tilde{V}_{k} & A_{k}+i \lambda \tilde{U}_{k} \\
-A_{k}+i \lambda \tilde{U}_{k} & i \lambda \tilde{V}_{k}
\end{array}\right] S_{k}=: T_{k} S_{k}
$$

Using Property 2' (i.e. $\Delta_{, t}=0$ ), the compatibility condition

$$
E \partial_{t}=\partial_{t} E
$$

where $E$ is the shift operator along the chain:

$$
E f_{k}=f_{k+1}
$$

applied to the linear problems (6) and (21), or (14) and (22), specifies $A_{k}$ in terms of the velocity:

$$
\begin{aligned}
A_{k} & =\frac{\lambda}{\sin (\lambda \Delta)}\left[\frac{2 q_{k}}{1+q_{k}^{2}} \tilde{V}_{k+1}+\frac{1-q_{k}^{2}}{1+q_{k}^{2}} \tilde{U}_{k+1}-\cos (\lambda \Delta) \tilde{U}_{k}\right]= \\
& =\frac{\lambda}{\sin (\lambda \Delta)}\left[\sin \varphi_{k} \tilde{V}_{k+1}+\cos \varphi_{k} \tilde{U}_{k+1}-\cos (\lambda \Delta) \tilde{U}_{k}\right]
\end{aligned}
$$


and yields the kinematics

$$
\begin{gathered}
2 q_{k, t}=-2 \cos (\lambda \Delta)\left(U_{k+1}+q_{k} V_{k+1}\right)+\left(1+q_{k}^{2}\right)\left[U_{k}+U_{k+2}+q_{k+1}\left(V_{k+1}+V_{k+2}\right)\right], \\
\left(1-q_{k}^{2}\right) V_{k+1}-\left(1+q_{k}^{2}\right) V_{k}=2 q_{k} U_{k+1} \quad\left(\cos \varphi_{k} V_{k+1}-V_{k}=\sin \varphi_{k} U_{k+1}\right),
\end{gathered}
$$

where

$$
V_{k}=\frac{\lambda}{\sin (\lambda \Delta)} \tilde{V}_{k}, \quad U_{k}=\frac{\lambda}{\sin (\lambda \Delta)} \tilde{U}_{k} .
$$

Using (27), equation (26) can be rewritten in the following convenient form

$$
2 q_{k, t}=-2 \cos (\lambda \Delta)\left(U_{k+1}+q_{k} V_{k+1}\right)+\mathcal{R}\left(U_{k+1}+q_{k} V_{k+1}\right)
$$

in terms of the recursion operator

$$
\mathcal{R}:=\left(1+q_{k}^{2}\right)\left[E+E^{-1}+2\left(q_{k+1} E-q_{k-1}\right)(E-1)^{-1} \frac{q_{k}}{1+q_{k}^{2}}\right]
$$

which is a real reduction of the recursion operator obtained in [16], associated with the AL spectral problem (11). The operator $(E-1)^{-1}$, formal inverse of $(E-1)$, takes the following explicit form:

$$
(E-1)^{-1} f_{k}=\sum_{j=k_{0}}^{k-1} f_{j}+b,
$$

where the choice of the constants $k_{0}$ and $b$ depends on the space of functions $f_{k}$ under consideration.

Equations (29) (27) show that the kinematics of the curve depends explicitly on the radius of the sphere through $\cos (\lambda \Delta)$; a drastic way to satisfy Property 3 ' is to choose the combination $\left(U_{k+1}+q_{k} V_{k+1}\right)$ as the real eigenfunction of the recursion operator $\mathcal{R}$ for the eigenvalue $2 \cos (\lambda \Delta)$, i.e.:

$$
U_{k+1}+q_{k} V_{k+1}=\Psi_{k}(\lambda),
$$

where

$$
\mathcal{R} \Psi_{k}(\lambda)=2 \cos (\lambda \Delta) \Psi_{k}(\lambda) .
$$

This velocity field, which generates the rigid motion $q_{k, t}=0$ of the discrete curve on the sphere of radius $1 / \lambda$, can be used to generate more complicated dynamics. For instance, if, on the sphere of radius $1 / \lambda$, one uses the velocity field which would give rise to the rigid motion on the sphere of radius $1 / \lambda_{0} \neq 1 / \lambda$, normalized in the following way

$$
U_{k+1}+q_{k} V_{k+1}=\frac{\Psi_{k}\left(\lambda_{0}\right)}{\cos \left(\lambda_{0} \Delta\right)-\cos (\lambda \Delta)},
$$

then one would satisfy Property 3', obtaining an example of integrable discrete dynamics with sources (with a singular dispersion relation):

$$
q_{k, t}=\Psi_{k}\left(\lambda_{0}\right) \Leftrightarrow\left[\mathcal{R}-2 \cos \left(\lambda_{0} \Delta\right)\right] q_{k, t}=0 .
$$

If, in particular, $\lambda_{0}=0$, then

$$
\overrightarrow{\mathbf{v}}_{k}=\frac{\sin (\lambda \Delta)}{\lambda[1-\cos (\lambda \Delta)]}\left(V_{k} \hat{\mathbf{t}}_{k}+U_{k} \hat{\mathbf{n}}_{k}\right)
$$

where

$$
\begin{aligned}
& U_{k+1}-U_{k}+q_{k}\left(V_{k+1}+V_{k}\right)=0, \\
& V_{k+1}-V_{k}-q_{k}\left(U_{k+1}+U_{k}\right)=0,
\end{aligned}
$$


and

$$
q_{k, t}=\Psi_{k}(0)=\frac{1}{2}\left(1+q_{k}^{2}\right)\left(U_{k+1}+U_{k}\right) \Leftrightarrow \varphi_{k, t}=U_{k+1}+U_{k} .
$$

Equations (37), satisfied by the velocity field which gives rise to the rigid motion on the plane, can be integrated to

$$
U_{k}=c \sin \Omega_{k}, \quad V_{k}=-c \cos \Omega_{k},
$$

where $\Omega_{k}$ is the (incomplete) total curvature:

$$
\Omega_{k}=\sum_{l}^{k-1} \varphi_{l},\left(\Omega_{k+1}-\Omega_{k}=\varphi_{k}\right) .
$$

Consequently equation (38) becomes the well-known discrete sine-Gordon (SG) equation [17] [6]:

$$
\varphi_{k, t}=c\left(\sin \Omega_{k+1}+\sin \Omega_{k}\right) \Leftrightarrow\left(\Omega_{k+1}-\Omega_{k}\right)_{, t}=c\left(\sin \Omega_{k+1}+\sin \Omega_{k}\right),
$$

which can also be written in the following form

$$
\left(u_{k+1}-u_{k}\right)_{, t}=c \sin \left(u_{k+1}+u_{k}\right)
$$

through the change of variables

$$
u_{k+1}+u_{k}=\Omega_{k}, \quad\left(u_{k}=\sum_{j}^{1} \varphi_{k-2 j}\right) .
$$

Using the well-known connection between discrete dynamics and Bäcklund transformations (BT) [18, we observe that equation (42) can be viewed as the celebrated BT of the SG equation $u, t x=\sin u$ [19], interpreting $u_{k}$ and $u_{k+1}$ as two different solutions of it. Therefore equation (41) has two different geometric meanings: i) it describes the integrable dynamics of a sequence of points on the sphere, subjected to a velocity field which would give rise to a rigid motion on the plane, and ii) it provides an elegant way to construct a new (pseudo)spherical surface from a given one.

The linear dependence on $\cos (\lambda \Delta)$ in equation (29) suggests that Property 3 ' can be satisfied also looking for velocity fields given by suitable Laurent expansions in powers of $\cos (\lambda \Delta)$ :

$$
\left[\begin{array}{c}
V_{k} \\
U_{k}
\end{array}\right]=\sum_{j=-m_{1}}^{m_{2}}(\cos (\lambda \Delta))^{j}\left[\begin{array}{c}
V_{k}^{(m-j)} \\
U_{k}^{(m-j)}
\end{array}\right], m_{1}, m_{2} \geq 0 .
$$

Substituting equation (44) into (29) and requiring independence of $\cos (\lambda \Delta)$, one obtains a class of integrable dynamics in the form

$$
g(\mathcal{R}) q_{k, t}=h(\mathcal{R})\left(1+q_{k}^{2}\right)\left(q_{k+1}-q_{k-1}\right),
$$

where $g$ and $h$ are arbitrary entire functions of their arguments. The simplest examples are the following: i) If $g=h=1$ we have

$$
\begin{gathered}
\overrightarrow{\mathbf{v}}_{k}=\frac{\sin (\lambda \Delta)}{\lambda}\left[\hat{\mathbf{t}}_{k}-q_{k-1} \hat{\mathbf{n}}_{k}\right], \\
q_{k, t}=\left(1+q_{k}^{2}\right)\left(q_{k+1}-q_{k-1}\right):=K_{k}^{(1)} \Leftrightarrow \varphi_{k, t}=\tan \left(\frac{\varphi_{k+1}}{2}\right)-\tan \left(\frac{\varphi_{k-1}}{2}\right) .
\end{gathered}
$$

In this case the velocity field $\overrightarrow{\mathbf{v}}_{k}$ admits the simple geometric construction shown in the figure below, in the case of the plane $(\lambda \rightarrow 0)$. 
ii) If $g=1$ and $h(x)=x$ we have

$$
\begin{gathered}
\overrightarrow{\mathbf{v}}_{k}=\frac{4 \sin (\lambda \Delta)}{\lambda}\left\{\left(\cos (\lambda \Delta)+q_{k} q_{k-1}\right) \hat{\mathbf{t}}_{k}+\left(-q_{k-1} \cos (\lambda \Delta)+\frac{1}{2}\left(1+q_{k-1}^{2}\right)\left(q_{k}-q_{k-2}\right)-q_{k-1}^{2} q_{k}\right) \hat{\mathbf{n}}_{k}\right\}, \\
q_{k, t}=\left(1+q_{k}^{2}\right)\left[\left(1+q_{k+1}^{2}\right) q_{k+2}-\left(1+q_{k-1}^{2}\right) q_{k-2}+q_{k}\left(q_{k+1}^{2}-q_{k-1}^{2}\right)\right]:=K_{k}^{(2)} .
\end{gathered}
$$

As it was observed in [10], the linear combination

$$
q_{k, t}=K_{k}^{(2)}-2 K_{k}^{(1)}
$$

of the first two flows reduces, in the continuous limit, to the $\mathrm{mKdV}$ equation

$$
\kappa_{, t^{\prime}}=\kappa_{, s s s}+\frac{3}{2} \kappa^{2} \kappa_{, s}, \quad t^{\prime}=2 \Delta^{3} t .
$$

iii) If $g(x)=x$ and $h=0$, then

$$
\begin{gathered}
\overrightarrow{\mathbf{v}}_{k}=\frac{\tan (\lambda \Delta)}{\lambda}\left[V_{k}^{(-1)} \hat{\mathbf{t}}_{k}+U_{k}^{(-1)} \hat{\mathbf{n}}_{k}\right], \\
q_{k, t}=-\frac{1}{2}\left(1+q_{k}^{2}\right)\left(U_{k+1}^{(-1)}-U_{k-1}^{(-1)}\right),
\end{gathered}
$$

where the normal velocity field satisfies the difference equation

$$
\left(q_{k+1}+\frac{1}{q_{k+1}}\right) U_{k+2}^{(-1)}+\left(q_{k}-\frac{1}{q_{k}}\right) U_{k+1}^{(-1)}-\left(q_{k+1}-\frac{1}{q_{k+1}}\right) U_{k}^{(-1)}-\left(q_{k}+\frac{1}{q_{k}}\right) U_{k-1}^{(-1)}=0
$$

and the tangent velocity

$$
2 V_{k}^{(-1)}=-\left(q_{k}+\frac{1}{q_{k}}\right) U_{k+1}^{(-1)}+\left(q_{k}-\frac{1}{q_{k}}\right) U_{k-1}^{(-1)} .
$$

We remark that this integrable dynamics corresponds to the velocity field

$$
U_{k+1}+q_{k} V_{k+1}=-\frac{\Psi_{k}\left(\lambda_{0}\right)}{\cos (\lambda \Delta)}
$$

which would give rise to a rigid motion on the sphere of radius $1 / \lambda_{0}=2 \Delta / \pi$.

\section{The motion of a discrete curve in $S^{3}(R)$}

\subsection{The discrete curve in $S^{3}(R)$}

The plane $\pi_{k}$ is, as in the previous Section, the oriented plane given by the ordered pair of vectors $\left(\overrightarrow{\mathbf{r}}_{k}, \overrightarrow{\mathbf{r}}_{k+1}\right)$. Similarly, the unit vectors $\hat{\mathbf{t}}_{k}$ and $\hat{\mathbf{t}}_{k}^{\prime}$ are tangent to the big circle passing through the points $\overrightarrow{\mathbf{r}}_{k}$ and $\overrightarrow{\mathbf{r}}_{k+1}$.

The tangent plane $\tau_{k}$ in the point $\overrightarrow{\mathbf{r}}_{k}$ to the discrete curve is defined by the two vectors $\hat{\mathbf{t}}_{k-1}^{\prime}$ and $\hat{\mathbf{t}}_{k}$. The angle of geodesic curvature $\varphi_{k}$ is the angle between the vectors $\hat{\mathbf{t}}_{k}^{\prime}$ and $\hat{\mathbf{t}}_{k+1}$. The orientation of the plane $\tau_{k+1}$ is choosen by the condition $0<\varphi_{k}<\pi$. The normal vector $\hat{\mathbf{n}}_{k}$ is the unit vector of $\tau_{k}$ orthogonal to $\hat{\mathbf{t}}_{k}$ and such that the pair $\left(\hat{\mathbf{t}}_{k}, \hat{\mathbf{n}}_{k}\right)$ gives correct orientation of the plane $\tau_{k}$.

The binormal vector $\hat{\mathbf{b}}_{k}=\hat{\mathbf{r}}_{k} \times \hat{\mathbf{t}}_{k} \times \hat{\mathbf{n}}_{k}$, together with the normal vector $\hat{\mathbf{n}}_{k}$, define the normal plane $\nu_{k}$ in point $\overrightarrow{\mathbf{r}}_{k}$. The orientation of the plane $\nu_{k}$ is given by the ordering $\left(\hat{\mathbf{n}}_{k}, \hat{\mathbf{b}}_{k}\right)$. We first remark that 
the vectors $\hat{\mathbf{r}}_{k+1}$ and $\hat{\mathbf{t}}_{k}^{\prime}$ span the plane $\pi_{k}$; moreover, since $\hat{\mathbf{t}}_{k}^{\prime} \in \tau_{k+1}$, it follows that $\hat{\mathbf{b}}_{k+1} \perp \hat{\mathbf{t}}_{k}^{\prime}$ which, together with the obvious condition $\hat{\mathbf{b}}_{k+1} \perp \hat{\mathbf{r}}_{k+1}$, imply that $\hat{\mathbf{b}}_{k+1} \in \nu_{k}=\pi_{k}^{\perp}$. The angle $\theta_{k}$ between the vectors $\hat{\mathbf{b}}_{k}$ and $\hat{\mathbf{b}}_{k+1}$ (which belong to the same plane $\nu_{k}$ ), measured according to the orientation of the plane $\nu_{k}$, is called the angle of (the geodesic) torsion of the curve in point $\overrightarrow{\mathbf{r}}_{k}$. In the case of $\mathbf{R}^{3}$ it is ilustrated on the figure below

In the continuous limit, the angle of torsion $\theta_{k}$ reduces to the familiar torsion $\tau(s)$ of the corresponding continuous curve in the following way:

$$
\frac{\theta_{k}}{\Delta} \stackrel{\Delta \rightarrow 0}{\longrightarrow} \tau(s)
$$

\subsection{The Hasimoto transformation and the spectral problem}

As in the case of the continuous curve, it is convenient to change the basis of the normal plane $\nu_{k}$ through a rotation of the angle $\sigma_{k-1}=(E-1)^{-1} \theta_{k}=\sum_{l}^{k-1} \theta_{l}$.

$$
\begin{aligned}
& \hat{\mathbf{n}}_{k}^{1}=\cos \sigma_{k-1} \hat{\mathbf{n}}_{k}-\sin \sigma_{k-1} \hat{\mathbf{b}}_{k}, \\
& \hat{\mathbf{n}}_{k}^{i}=\sin \sigma_{k-1} \hat{\mathbf{n}}_{k}+\cos \sigma_{k-1} \hat{\mathbf{b}}_{k} .
\end{aligned}
$$

This change of basis corresponds to a partial "integration" of the Frenet equations in the normal plane, since the vectors $\hat{\mathbf{n}}_{k}^{1}, \hat{\mathbf{n}}_{k}^{i}$ do not vary from the point of view of the normal plane (this is the discrete analog of the parallel transport in the normal bundle).

As in the case of a continuous curve, it is convenient to interprete any vector of the normal plane $\nu_{k}$ as the complex number

$$
\vec{\phi}_{k}=\operatorname{Re} \phi_{k} \hat{\mathbf{n}}_{k}^{1}+\operatorname{Im} \phi_{k} \hat{\mathbf{n}}_{k}^{i} \Leftrightarrow \phi_{k}=\operatorname{Re} \phi_{k}+i \operatorname{Im} \phi_{k} .
$$

The transition from the basis $\left(\hat{\mathbf{r}}_{k}, \hat{\mathbf{t}}_{k}, \hat{\mathbf{n}}_{k}^{1}, \hat{\mathbf{n}}_{k}^{i}\right)$ to the basis $\left(\hat{\mathbf{r}}_{k+1}, \hat{\mathbf{t}}_{k+1}, \hat{\mathbf{n}}_{k+1}^{1}, \hat{\mathbf{n}}_{k+1}^{i}\right)$ can be obtained by the superposition of the following five rotations:

i) rotation in the plane $\nu_{k}$ of the angle $\sigma_{k-1}$

$$
\left(\hat{\mathbf{r}}_{k}, \hat{\mathbf{t}}_{k}, \hat{\mathbf{n}}_{k}^{1}, \hat{\mathbf{n}}_{k}^{i}\right) \longrightarrow\left(\hat{\mathbf{r}}_{k}, \hat{\mathbf{t}}_{k}, \hat{\mathbf{n}}_{k}, \hat{\mathbf{b}}_{k}\right)
$$

ii) rotation in the plane $\pi_{k}$ of the angle $2 \alpha=\lambda \Delta$

$$
\left(\hat{\mathbf{r}}_{k}, \hat{\mathbf{t}}_{k}, \hat{\mathbf{n}}_{k}, \hat{\mathbf{b}}_{k}\right) \longrightarrow\left(\hat{\mathbf{r}}_{k+1}, \hat{\mathbf{t}}_{k}^{\prime}, \hat{\mathbf{n}}_{k}, \hat{\mathbf{b}}_{k}\right)
$$

iii) rotation in the plane $\nu_{k}$ of the angle $\theta_{k}$

$$
\left(\hat{\mathbf{r}}_{k+1}, \hat{\mathbf{t}}_{k}^{\prime}, \hat{\mathbf{n}}_{k}, \hat{\mathbf{b}}_{k}\right) \longrightarrow\left(\hat{\mathbf{r}}_{k+1}, \hat{\mathbf{t}}_{k}^{\prime}, \hat{\mathbf{n}}_{k}^{\prime}, \hat{\mathbf{b}}_{k+1}\right)
$$

iv) rotation in the plane $\tau_{k+1}$ of the angle $\varphi_{k}$

$$
\left(\hat{\mathbf{r}}_{k+1}, \hat{\mathbf{t}}_{k}^{\prime}, \hat{\mathbf{n}}_{k}^{\prime}, \hat{\mathbf{b}}_{k+1}\right) \longrightarrow\left(\hat{\mathbf{r}}_{k+1}, \hat{\mathbf{t}}_{k+1}, \hat{\mathbf{n}}_{k+1}, \hat{\mathbf{b}}_{k+1}\right)
$$

v) rotation in the plane $\nu_{k+1}$ of the angle $-\sigma_{k}$

$$
\left(\hat{\mathbf{r}}_{k+1}, \hat{\mathbf{t}}_{k+1}, \hat{\mathbf{n}}_{k+1}, \hat{\mathbf{b}}_{k+1}\right) \longrightarrow\left(\hat{\mathbf{r}}_{k+1}, \hat{\mathbf{t}}_{k+1}, \hat{\mathbf{n}}_{k+1}^{1}, \hat{\mathbf{n}}_{k+1}^{i}\right) .
$$


Rotations in $\mathbf{R}^{4}$ can be represented in a similar form as in the previous Section. A fixed Cartesian basis in $\mathbf{R}^{4}$ can be identified with the following $2 \times 2$ matrices $\mathcal{E}=\left(\varepsilon_{0}=\mathrm{Id}, \varepsilon_{1}=i \sigma_{3}, \varepsilon_{2}=i \sigma_{1}, \varepsilon_{3}=-i \sigma_{2}\right)$.

$$
\overrightarrow{\mathbf{v}} \in \mathbf{R}^{4} \Leftrightarrow \sigma(\overrightarrow{\mathbf{v}})=\sum_{l=0}^{3} \mathrm{v}^{l} \varepsilon_{l} ; \quad\|\overrightarrow{\mathbf{v}}\|^{2}=\operatorname{det} \sigma(\overrightarrow{\mathbf{v}}) .
$$

Any rotation $O$ can be represented in terms of two matrices $P$ and $S$ of the $S U(2)$ group in the following way

$$
\overrightarrow{\mathbf{w}}=O \overrightarrow{\mathbf{v}} \Leftrightarrow \sigma(\overrightarrow{\mathbf{w}})=P^{-1} \sigma(\overrightarrow{\mathbf{v}}) S .
$$

When we are given a sequence of orthonormal frames $\mathcal{F}_{k}=\left(\mathbf{e}_{k}, \mathbf{f}_{k}, \mathbf{g}_{k}, \mathbf{h}_{k}\right)$ and the corresponding matrices $P_{k}, S_{k}$

$$
\mathcal{F}_{k}=P_{k}^{-1} \mathcal{E} S_{k}
$$

then we define matrices $A_{k}$ and $B_{k}$ by the relations $S_{k+1}=A_{k} S_{k}$ and $P_{k+1}=B_{k} P_{k}$; moreover

$$
P_{k} \mathcal{F}_{k+1} S_{k}^{-1}=B_{k}^{-1} \mathcal{E} A_{k}
$$

If the rotation takes place in the space perpendicular to the first vector of the basis $\mathcal{F}_{k}$ (i.e. $\mathbf{e}_{k+1}=\mathbf{e}_{k}$ ) then $A_{k}=B_{k}$. For example the matrices

$$
A_{k}=B_{k}=\left[\begin{array}{cc}
\mathrm{e}^{-i \theta / 2} & 0 \\
0 & \mathrm{e}^{i \theta / 2}
\end{array}\right]
$$

represent the rotation in the plane $\left(\mathbf{g}_{k}, \mathbf{h}_{k}\right)$ of the angle $\theta$, and the matrices

$$
A_{k}=B_{k}=\left[\begin{array}{cc}
\cos (\varphi / 2) & \sin (\varphi / 2) \\
-\sin (\varphi / 2) & \cos (\varphi / 2)
\end{array}\right]
$$

represent the rotation in the plane $\left(\mathbf{f}_{k}, \mathbf{g}_{k}\right)$ of the angle $\varphi$.

We will also need the representation of the rotation in the plane $\left(\mathbf{e}_{k}, \mathbf{f}_{k}\right)$ of the angle $2 \alpha$, given by matrices

$$
A_{k}=\left[\begin{array}{cc}
\mathrm{e}^{i \alpha} & 0 \\
0 & \mathrm{e}^{-i \alpha}
\end{array}\right], B_{k}=\left[\begin{array}{cc}
\mathrm{e}^{-i \alpha} & 0 \\
0 & \mathrm{e}^{i \alpha}
\end{array}\right] .
$$

We are now in the position to calculate the transition matrices when the sequence of the orthonormal frames is the sequence of the frames along the discrete curve $\mathcal{F}_{k}=\left(\hat{\mathbf{r}}_{k}, \hat{\mathbf{t}}_{k}, \hat{\mathbf{n}}_{k}^{1}, \hat{\mathbf{n}}_{k}^{i}\right)$. The corresponding spin matrices $P_{k}, S_{k}$ are subjected to the relations

$$
\begin{aligned}
P_{k+1} & =\left[\begin{array}{cc}
\mathrm{e}^{-i \alpha} \cos \left(\varphi_{k} / 2\right) & \mathrm{e}^{i \alpha} \sin \left(\varphi_{k} / 2\right) \mathrm{e}^{i \sigma_{k}} \\
-\mathrm{e}^{-i \alpha} \sin \left(\varphi_{k} / 2\right) \mathrm{e}^{-i \sigma_{k}} & \mathrm{e}^{i \alpha} \cos \left(\varphi_{k} / 2\right)
\end{array}\right] P_{k} \\
& =\frac{1}{\sqrt{1+\left|q_{k}\right|^{2}}}\left[\begin{array}{cc}
\zeta^{-1} & \zeta q_{k} \\
-\zeta^{-1} \bar{q}_{k} & \zeta
\end{array}\right] P_{k}=: B_{k} P_{k}, \\
S_{k+1} & =\left[\begin{array}{cc}
\mathrm{e}^{i \alpha} \cos \left(\varphi_{k} / 2\right) & \mathrm{e}^{-i \alpha} \sin \left(\varphi_{k} / 2\right) \mathrm{e}^{i \sigma_{k}} \\
-\mathrm{e}^{i \alpha} \sin \left(\varphi_{k} / 2\right) \mathrm{e}^{-i \sigma_{k}} & \mathrm{e}^{-i \alpha} \cos \left(\varphi_{k} / 2\right)
\end{array}\right] S_{k} \\
& =\frac{1}{\sqrt{1+\left|q_{k}\right|^{2}}}\left[\begin{array}{cc}
\zeta & \zeta^{-1} q_{k} \\
-\zeta \bar{q}_{k} & \zeta^{-1}
\end{array}\right] S_{k}=: A_{k} S_{k},
\end{aligned}
$$

where

$$
q_{k}=\tan \left(\varphi_{k} / 2\right) \mathrm{e}^{i \sigma_{k}}, \quad \zeta=\mathrm{e}^{i \alpha}
$$


We remark that the transformation (69) between the curvature and torsion angles and the complex field $q_{k}$ is the discrete analogue of the Hasimoto transformation, introduced for continuous curves in [20]. We also remark that $B_{k}=A_{k} \operatorname{diag}\left[\zeta^{-2}, \zeta^{2}\right]$.

As before the linear problem can be transformed into the AL spectral problem:

$$
\tilde{S}_{k+1}=\left[\begin{array}{cc}
\zeta & q_{k} \\
-\bar{q}_{k} & \zeta^{-1}
\end{array}\right] \tilde{S}_{k}, \quad \tilde{P}_{k+1}=\left[\begin{array}{cc}
\zeta^{-1} & q_{k} \\
-\bar{q}_{k} & \zeta
\end{array}\right] \tilde{P}_{k}
$$

(but now in the reduction $r_{k}=-\bar{q}_{k}$ ) relaxing the conditions $\operatorname{det} S_{k}=\operatorname{det} P_{k}=1$ (but keeping still $\left.\operatorname{det} S_{k}=\operatorname{det} P_{k}\right)$ and changing the basis $\left(\hat{\mathbf{r}}_{k}, \hat{\mathbf{t}}_{k}\right)$ of the plane $\pi_{k}$ to the new basis $\left(\hat{\mathbf{r}}_{k}^{\prime}, \hat{\mathbf{a}}_{k}\right)$.

\subsection{The integrable motions in $S^{3}(R)$}

Proceeding as in Section 2.3 we have that

$$
\begin{gathered}
\overrightarrow{\mathbf{r}}_{k, t}=\frac{\sin (\lambda \Delta)}{\lambda}\left(V_{k} \hat{\mathbf{t}}_{k}+\vec{\phi}_{k}\right), \\
\vec{\phi}_{k}=\operatorname{Re} \phi_{k} \hat{\mathbf{n}}_{k}^{1}+\operatorname{Im} \phi_{k} \hat{\mathbf{n}}_{k}^{i}=\operatorname{Re}\left(\phi_{k} \mathrm{e}^{-i \sigma_{k-1}}\right) \hat{\mathbf{n}}_{k}+\operatorname{Im}\left(\phi_{k} \mathrm{e}^{-i \sigma_{k-1}}\right) \hat{\mathbf{b}}_{k}
\end{gathered}
$$

and, consequently,

$$
\begin{gathered}
S_{k, t}=T_{k} S_{k}, \\
T_{k}=\frac{i}{2}\left[\begin{array}{cc}
\gamma_{k} & \delta_{k} \\
\bar{\delta}_{k} & -\gamma_{k}
\end{array}\right] \in s u(2) .
\end{gathered}
$$

The compatibility condition between equations (68)(71) and (72) specifies the entries $\gamma_{k}$ and $\delta_{k}$ in terms of the velocity field:

$$
\begin{aligned}
\delta_{k} & =i \zeta^{-2} \phi_{k}-i\left(\phi_{k+1}+q_{k}\left(V_{k}+V_{k+1}\right)\right) \\
\gamma_{k} & =W_{k}+\sin (\lambda \Delta) V_{k} \\
W_{k+1}-W_{k} & =-\operatorname{Re}\left(i \bar{q}_{k}\left[\phi_{k+2}-\phi_{k}+q_{k+1}\left(V_{k+1}+V_{k+2}\right)\right]\right)
\end{aligned}
$$

and yields the kinematics

$$
\begin{aligned}
2 q_{k, t}= & -2 \cos (\lambda \Delta)\left(\phi_{k+1}+q_{k} V_{k+1}\right)+\left(1+\left|q_{k}\right|^{2}\right)\left[\phi_{k}+\phi_{k+2}+q_{k+1}\left(V_{k+1}+V_{k+2}\right)\right] \\
+ & 2 i q_{k} W_{k}+q_{k}\left(q_{k} \bar{\phi}_{k}-\bar{q}_{k} \phi_{k}\right) \\
= & -2 \cos (\lambda \Delta)\left(\phi_{k+1}+q_{k} V_{k+1}\right)+\mathcal{R}\left(\phi_{k+1}+q_{k} V_{k+1}\right), \\
& \left(1-\left|q_{k}^{2}\right|\right) V_{k+1}-\left(1+\left|q_{k}\right|^{2}\right) V_{k}=q_{k} \bar{\phi}_{k+1}+\bar{q}_{k} \phi_{k+1},
\end{aligned}
$$

where

$$
\begin{aligned}
\mathcal{R} f_{k} & :=\left(1+\left|q_{k}\right|^{2}\right)\left(f_{k+1}+f_{k-1}+2\left(q_{k+1} E-q_{k-1}\right)(E-1)^{-1} \operatorname{Re} \frac{\bar{q}_{k} f_{k}}{1+\left|q_{k}\right|^{2}}\right) \\
& -2 i q_{k}(E-1)^{-1} \operatorname{Im}\left(\bar{q}_{k+1} f_{k}-\bar{q}_{k} f_{k+1}\right) .
\end{aligned}
$$

Substituting the ansatz

$$
\left[\begin{array}{l}
V_{k} \\
\phi_{k}
\end{array}\right]=\sum_{j=0}^{m}(\cos (\lambda \Delta))^{j}\left[\begin{array}{l}
V_{k}^{(m-j)} \\
\phi_{k}^{(m-j)}
\end{array}\right]
$$


into equation (74) and requiring independence of $\cos (\lambda \Delta)$, we finally obtain the following class of integrable dynamics:

$$
q_{k, t}=h_{0}(\mathcal{R})\left(1+\left|q_{k}\right|^{2}\right)\left(q_{k+1}-q_{k-1}\right)+h_{1}(\mathcal{R})\left(i q_{k}\right),
$$

where $h_{0}$ and $h_{1}$ are arbitrary entire functions with real coefficients.

We remark that, in the degenerate case of the curve on $S^{2}\left(q_{k} \in \mathbf{R}\right)$ we are forced to choose $h_{1}=0$ and only the first hierarchy survives.

We also remark that equation (74) implies the following interesting connection:

$$
K_{k}^{(m)}=\phi_{k+1}^{(m)}+q_{k} V_{k+1}^{(m)}
$$

between the integrable commuting flows

$$
K_{k}^{(m)}=\mathcal{R}^{m-1}\left(1+\left|q_{k}\right|^{2}\right)\left(q_{k+1}-q_{k-1}\right) \text { and/or } K_{k}^{(m)}=\mathcal{R}^{m}\left(i q_{k}\right), \quad m \geq 0
$$

and the velocity fields. In the continuous limit this reduces to the result of [21].

The simplest examples are the following:

i) If $h_{0}=1, h_{1}=0$, then

$$
\begin{gathered}
\overrightarrow{\mathbf{v}}_{k}=\frac{\sin (\lambda \Delta)}{\lambda}\left(\hat{\mathbf{t}}_{k}-\overrightarrow{\mathbf{q}}_{k-1}\right)=\frac{\sin (\lambda \Delta)}{\lambda}\left(\hat{\mathbf{t}}_{k}-\left|q_{k-1}\right| \hat{\mathbf{n}}_{k}\right), \\
q_{k, t}=\left(1+\left|q_{k}\right|^{2}\right)\left(q_{k+1}-q_{k-1}\right)=: K_{k}^{(1)} .
\end{gathered}
$$

ii) If $h_{0}(x)=x, h_{1}=0$, then

$$
\begin{gathered}
\overrightarrow{\mathbf{v}}_{k}=\frac{4 \sin (\lambda \Delta)}{\lambda}\left\{\left(\cos (\lambda \Delta)+\frac{1}{2}\left(q_{k} \bar{q}_{k-1}+\bar{q}_{k} q_{k-1}\right)\right) \hat{\mathbf{t}}_{k}+\vec{\phi}_{k}\right\} \\
\phi_{k}=\left(-q_{k-1} \cos (\lambda \Delta)+\frac{1}{2}\left[\left(1+\left|q_{k-1}\right|^{2}\right)\left(q_{k}-q_{k-2}\right)-q_{k-1}\left(q_{k} \bar{q}_{k-1}+\bar{q}_{k} q_{k-1}\right)\right]\right) \\
q_{k, t}=K_{k}^{(2)}:= \\
\left(1+\left|q_{k}\right|^{2}\right)\left[\left(1+\left|q_{k+1}\right|^{2}\right) q_{k+2}-\left(1+\left|q_{k-1}\right|^{2}\right) q_{k-2}+\bar{q}_{k}\left(q_{k+1}^{2}-q_{k-1}^{2}\right)+q_{k}\left(q_{k+1} \bar{q}_{k-1}-q_{k-1} \bar{q}_{k+1}\right)\right] .
\end{gathered}
$$

The linear combination

$$
q_{k, t}=K_{k}^{(2)}-2 K_{k}^{(1)}
$$

reduces, in the continuous limit, to the complex mKdV equation

$$
\psi_{, t^{\prime}}=\psi_{, s s s}+\frac{3}{2}|\psi|^{2} \psi_{, s}, \quad \psi(s):=\lim _{\Delta \rightarrow 0} \frac{2 q_{k}}{\Delta}=\kappa(s) \mathrm{e}^{i \partial_{s}^{-1} \tau}, t^{\prime}=2 \Delta^{3} t .
$$

iii) If $h_{0}=0$ and $h_{1}(x)=x$, we obtain

$$
\begin{gathered}
\overrightarrow{\mathbf{v}}_{k}=\frac{2 \sin (\lambda \Delta)}{\lambda}\left(\overrightarrow{\mathbf{i q}}_{k-1}\right)=\frac{2 \sin (\lambda \Delta)}{\lambda} \tan \left(\frac{\varphi_{k-1}}{2}\right) \hat{\mathbf{b}}_{k}, \\
q_{k, t}=i\left(1+\left|q_{k}\right|^{2}\right)\left(q_{k+1}+q_{k-1}\right) .
\end{gathered}
$$

The following combination of equation (88) with the "zero order flow" $q_{k, t}=i q_{k}$ :

$$
q_{k, t}=i\left[q_{k+1}-2 q_{k}+q_{k-1}+\left|q_{k}\right|^{2}\left(q_{k+1}+q_{k-1}\right)\right]
$$


reduces [10], in the continuous limit, to the NLS equation

$$
i \psi_{, t}=\psi_{, s s}+\frac{1}{2}|\psi|^{2} \psi, t^{\prime}=-\Delta^{2} t
$$

which describes the motion of a vortex filament in the localized induction approximation [20] 22]. We remark that, in this approximation, the velocity field which governs the motion of the vortex depends on its curvature $\kappa$ through the relation

$$
\overrightarrow{\mathbf{r}}_{, t}=\kappa \hat{\mathbf{b}}
$$

therefore since equation (89) has in $\mathbf{R}^{3}$ a velocity field of the same type:

$$
\overrightarrow{\mathbf{r}}_{k, t}=2 \Delta \tan \left(\frac{\varphi_{k-1}}{2}\right) \hat{\mathbf{b}}_{k}
$$

we expect it to be a good candidate for describing the motion of a discrete vortex in the same approximation.

\section{Acknowledgements}

We would like to thank A. B. Shabat who encouraged this research, A. Sym for pointing out ref. 114 and D. Levi and M. Bruschi for useful discussions.

\section{References}

[1] A. Doliwa and P. M. Santini, Phys. Lett. A 185 (1994) 373.

[2] B. A. Dubrovin, Func. Anal. Appl. 11 (1981) 265.

P. Painlevé, Bull. Soc. Math. Fr. 28(1900) 214.

[3] S. Novikov, S. V. Manakov, L. P. Pitaevskii and V. E. Zakharov, Theory of Solitons, The inverse Scattering Method, Contemporary Soviet Mathematics, New York: Consultant Bureau, 1984.

M. J. Ablowitz and H. Segur, Solitons and the Inverse Scattering Transform, Studies in Applied Mathematics 4, Philadelphia, PA: SIAM, 1981.

[4] B.A. Dubrovin, A.S. Fokas and P.M. Santini, Integrable Functional Equations and Algebraic Geometry, Preprint INS 208 1993, Invent. Math. (in press).

[5] R. Hirota, J. Phys. Soc. Jap. 43:6 (1977) 2079.

[6] D. Levi, O. Ragnisco and M. Bruschi, Nuovo Cimento 58 A (1980) 56.

L. Pilloni and D. Levi, Phys. Lett. 92 A (1982) 5.

[7] G. R. W. Quispel, F. W. Nijhoff, H. W. Capel and J. van der Linden, Physica 125 A, (1984) 344. F. W. Nijhoff, H. W. Capel, G. L. Wiersma and G. R. W. Quispel, Phys. Lett. 103 A (1984) 293.

V. G. Papageorgiu, F. W. Nijhoff and H. W. Capel, Phys. Lett. 147 A (1990) 106.

[8] A. S. Fokas, A. R. Its and A. V. Kitaev, Commun. Math. Phys. 142 (1991) 313.

[9] A. B. Shabat, Inverse Problems 6 (1992) 303.

[10] M. J. Ablowitz and J. F. Ladik, J. Math. Phys. 16 (1975) 598.

M. J. Ablowitz and J. F. Ladik, J. Math. Phys. 17 (1976) 1011.

M. J. Ablowitz, Stud. Appl. Math. 58 (1978) 17. 
[11] M. J. Ablowitz, D. J. Kaup, A. C. Newell and H. Segur, Stud. Appl. Math. 53 (1974) 249.

[12] W. Wunderlich, Ak. Wiss. Wien 160 (1951) 39.

M. Melko and U. Pinkall, Lattice models of pseudospherical surfaces in $\mathbf{R}^{3}$, unpublished paper.

[13] U. Pinkall, Discrete Harmonic Maps and Integrable Systems, lecture given at the conference Harmonic Maps and Integrable Systems, Leeds 1992

[14] J. A. Aminov, Differential geometry and topology of curves, Moscov, Nauka, 1987 (in Russian).

[15] P. Budinich and A. Trautman, The Spinorial Chessboard, Springer, Berlin, 1988.

H. B. Lawson and M. L. Michelson, Spin geometry, Princeton U. P., Princeton, 1989.

[16] S. C. Chiu and J. F. Ladik, J. Math. Phys. 18 (1977) 690.

[17] S. J. Orfanidis, Phys. Rev. 18 D, (1978) 3828.

[18] D. Levi and R. Benguria, Proc. Nat. Acad. Sci. (USA) 77 (1980) 5025.

[19] A. V. Bäcklund, Concerning Surfaces with Constant Negative Curvature New Era Printing Co. Lancaster, 1905.

[20] H. Hasimoto, J. Fluid Mech. 51 (1972) 477.

[21] J. Langer and R. Perline, J. Nonlin. Sci. 1 (1991) 71.

[22] G. K. Batchelor, An Introduction to Fluid Mechanics, Cambridge University Press, New York, 1967. 


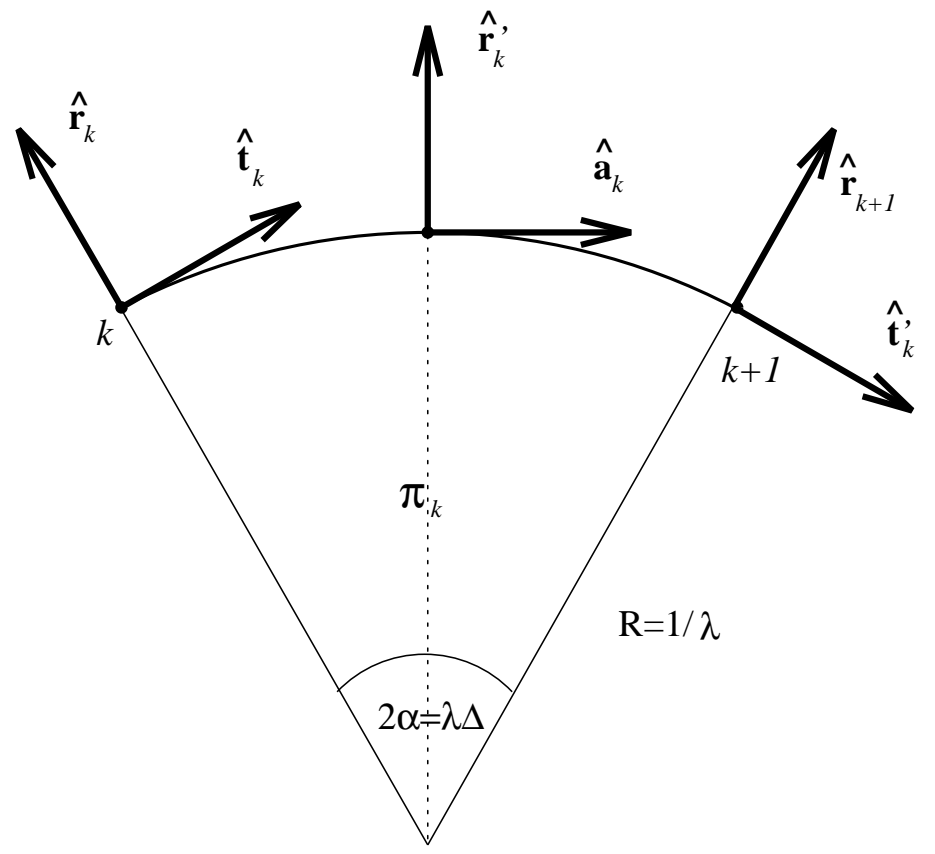

Fig. 1 


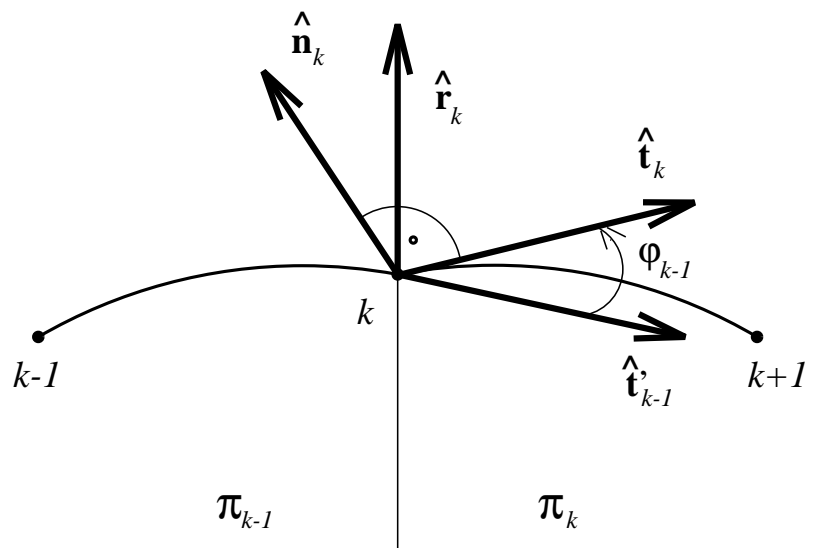

Fig. 2 
Fig. 3

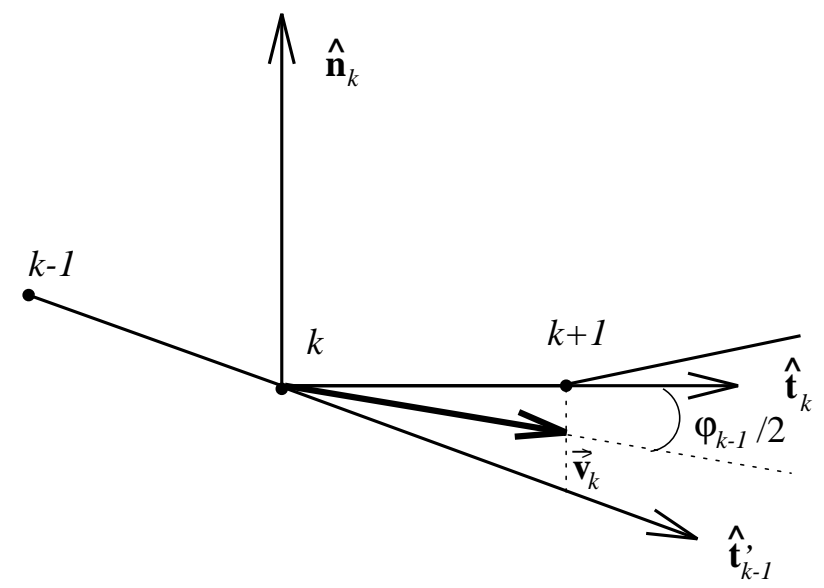




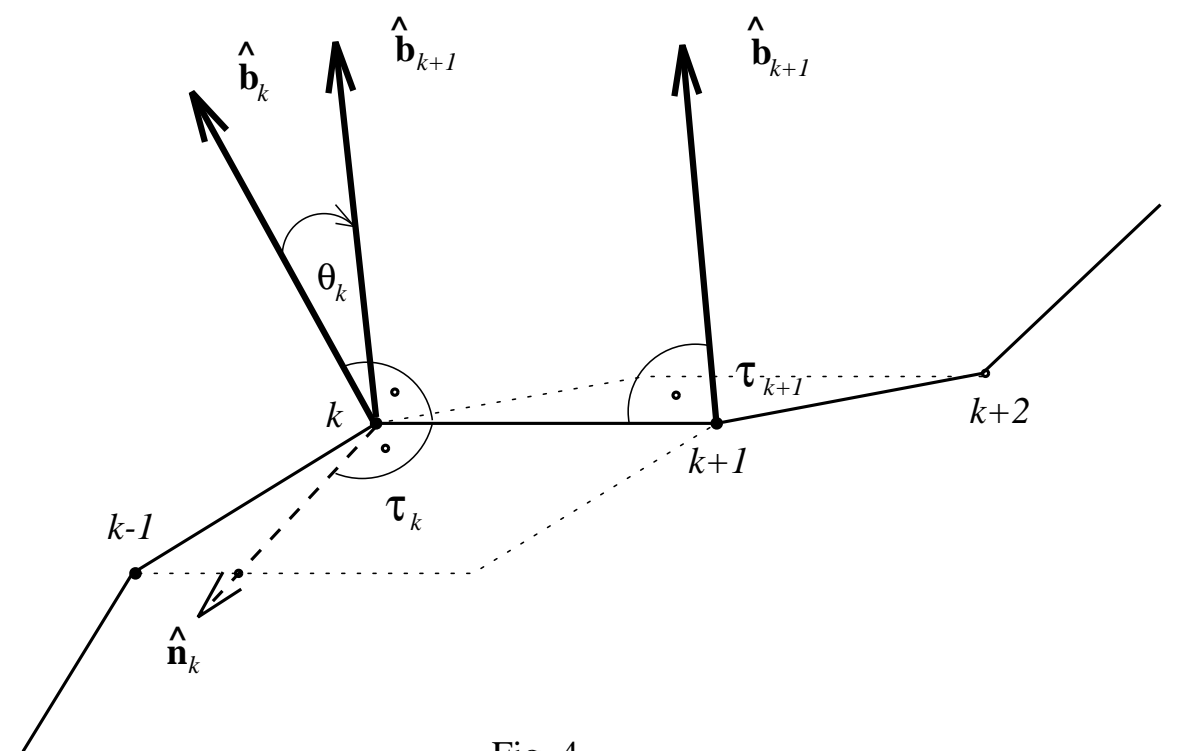

Fig. 4 